\title{
Efficient User Pairing for Performance Enhancement of Downlink NOMA Systems
}

\author{
Fahad Alraddady*
}

\begin{abstract}
Department of Computer Engineering, College of Computers and Information Technology, Taif University, Taif, 21944, Saudi Arabia *Corresponding Author: Fahad Alraddady. Email: alraddady@tu.edu.sa Received: 13 July 2021; Accepted: 17 September 2021
\end{abstract}

\begin{abstract}
In this paper, the resource allocation problem for user pairing (UP) in downlink non-orthogonal multiple access (NOMA) systems is investigated. NOMA allows the use of one subcarrier for more than one user at the same time, thus increases the total capacity of the wireless communication system. However, users pairing is a challenging task in the NOMA systems, because a good channel quality subcarrier should be selected and allocated for the user to enhance the performance of NOMA systems. The proposed UP algorithm aims to enhance the sum rate of the paired users per subcarrier and consequently enhance the total sum rate of downlink NOMA systems. Moreover, the proposed UP algorithm target to improve the fairness of the users. The proposed UP algorithm is based on a simple search for the subcarrier with the minimum average channel gains to be assigned its paired users and then excluding it from the next searching process. The proposed scheme ensures the higher channel gain for users by giving the priority to the subcarrier with the minimum average channel gains during the user pairing process. The simulation results demonstrate that the proposed UP algorithm can not only enhance the total sum rate compared with the random UP and conventional UP but also can enhance the fairness of the users. Moreover, it is clearly seen that the proposed UP algorithm provides the lowest outage probability.
\end{abstract}

Keywords: 5G; NOMA; sum-rate; user pairing; fairness

\section{Introduction}

The ongoing exciting development of smartphones and tablets has led these devices to play a crucial role in our daily lives. Thanks to a handful of services and applications offered in these devices, phone calls are no longer the sole application. Applications like watching the television, playing online video games, and taking part in online classes can be executed effectively through our smartphones. These increments in remote gadgets coupled with a fast insurgency in mixed media applications are changing the nature of wireless communication activity by increasingly requesting high data rates. Moreover, the growing importance of the Internet of Things (IoT) acquaints the need to associate each individual and each item [1]. Future radio access is trending towards developing 5G technology to satisfy those mentioned above and inconceivably high client information rates and system capacity requirements [2].

This work is licensed under a Creative Commons Attribution 4.0 International License, which permits unrestricted use, distribution, and reproduction in any medium, provided the original work is properly cited. 
One of the wireless networking techniques, multiple access, has a major influence on the use of the existing spectrum resources, system latency, and throughput. Multiple access signifies a strategy where a wireless channel is shared by numerous users to create a communication connection with a base station (BS) in the form of cellular radio. In an orthogonal multiple access (OMA) scheme, the clients access the resources symmetrical in nature, and the clients do not meddle with each other while they share the correspondence resource [3]. The minimal complexity and consequently the reduced implementation cost of receiver devices are the main advantages of exploiting OMA. On the other hand, the critical constraint of OMA is its strictly restricted ability to support many users or connections. As a result, spectral inefficiency will occur, and it would be impossible to endorse large-scale networking and IoT, both of which have been considered as the main features of $5 \mathrm{G}$ networks [4]. To overcome this limitation, NTT DoCoMo researchers innovated a new multiple access technique which is called non-orthogonal multiple access (NOMA), for the next generation (5G) mobile network [5]. NOMA is seen as one of the competitor access innovations for $5 \mathrm{G}$ remote portable correspondence to accomplish manifold capacity gains as a result of its high spectral efficiency [6]. NOMA allows multiple users with diverse channel conditions to share the same subcarrier, resulting in significant spectral efficiency improvements. Furthermore, NOMA can quickly support users with varying channel conditions, allowing it to meet the strict 5G criteria of ultra-high connectivity and ultra-low latency [7].

In this paper, the power-domain NOMA with respect to user pairing (UP) is being studied. Traditional power allocation (PA) strategies give clients with strong channel conditions more power. In contrast to these traditional schemes, the amount of power allocated to a user in power domain NOMA is set by its channel gain [8]; a user with a better channel condition is often given a lower power level. At the transmitter side, multiple users' signals are superimposed, and at the receiver side, successive interference cancellation (SIC) is utilized to decode the signal information sequentially until the aspired user's signal is attained [9]. The consumer with the stronger channel state, on the other hand, must first detect its partner's message, then deduct this information from its observation, and lastly interpret its own data. SIC and user pairing (UP) has a major influence on NOMA performance [10].

This study endeavors to examine the problem of user pairing (UP) in a downlink NOMA framework. The remainder sections are arranged in the following manner. Section 2 is a survey of related works. The system model is covered in Section 3. In Section 4, the proposed user pairing algorithm. The simulation results are demonstrated in Section 5, and the conclusion is included in Section 6.

\section{Related Works}

The principles of power-domain NOMA in both uplink and downlink conditions with single and multiple antennas were discussed in [8]. The authors explored the fundamentals of code-domain NOMA thoroughly. In addition, the article address power allocation (PA), user pairing (UP), and cooperative NOMA, in their basic form. Various opportunities and difficulties regarding the compatibility of NOMA with heterogeneous networks were also examined. A methodical approach to the newly emerging NOMA technology is provided in [9], from its use in conjunction with multiple-input, multiple-output (MIMO) technologies to the interplay between NOMA and cognitive radio as well as cooperative NOMA. The article also explores the standardization efforts surrounding the deployment of NOMA in LTE and 5G networks.

In [11], a solution for combined PA and user selection in NOMA systems for Stackelberg game-based revenue maximization is explored. The Lagrange multiplier technique is used in the conversion of the revenue maximization problem, and a univariate iterative procedure is utilized to find the optimal solution. The algorithm exhibits low complexity, and to assess the performance degradation, an outage analysis is presented in the condition that CSI is not perfect. The authors of [12] examined the systemlevel performance of NOMA along with SIC technology on the receiver side. The article demonstrates 
that under multiple sets of configurations that NOMA outperforms OMA overall system performance. The comparison was over the key link adaptation functionalities that include hybrid automatic repeat request (HARQ), adaptive modulation and coding (AMC), time/frequency-domain scheduling, and functionalities unique to NOMA, such as dynamic multiuser PA.

Fair-NOMA is introduced in [13] in which each user is given a portion of the transmitted subcarrier power so that their capacity is always equal to or more than the capacity attained by OMA. It is demonstrated that the capacity of Fair-NOMA with $K$ users can always be enhanced for each additional user to the network. For the downlink NOMA system, two subcarrier-user assignment algorithms (SUAAs) are presented in [14] to improve spectral efficiency, outage probability, fairness, and weak users' data rate. Two types of SUAA are proposed SUAA. Regarding arrangement order in the first proposed SUAA, to prevent selecting a user who has the least channel gain with any subcarrier, begin with the worst subcarrier first (WSF). In the second proposed SUAA, arrangement order follows spectral efficiency maximization (SEM) and has high computational complexity. It is shown in the paper that the two advocated SUAAs can gain a tremendous boost in the spectral efficiency, outage probability, user fairness and, weak user data rate compared to the other SUAA algorithms.

With the goal to enhance the proportional fairness of the users in the NOMA uplink communication systems, UP and PA techniques that work together are suggested in [15]. The article explores two different scenarios, basic and complex. In the PA part, the near-optimal PA solution for each type of user pair can be obtained in a variety of methods. A probability-based Tabu search strategy is presented in the UP part of the basic scenario to figure out the near-optimal UP solution. In the complex scenario, the optimization problem in PA part is a stochastic programming problem with an outage rate constraint. A particle swarm optimization technique based on prediction is utilized to provide a solution to the stochastic programming problem.

A Power Allocation technique is introduced from information rate expression examination of NOMA [16]. NOMA users can get rates in the frequency domain schemes that are equal to or higher than those attained with Orthogonal Multiple Access (OMA) using this method. The results obtained from computer system-level simulations showed that the proposed technique boosts the mean cell spectral efficiency while maintaining a decent level of fairness in the distribution of resources across users within a cell.

In [17], the challenges of user scheduling and resource allocation for a NOMA downlink network that is characterized by a base station allocating spectrum and power assets to a number of users were investigated. The authors of the paper aimed to optimize both PA and sub-channel assignment in order to maximize the weighted total sum rate while also keeping user fairness in mind. A matching technique is devised for the sub-channel allocation problem that converges after a narrow number of iterations to a two-side exchange stable matching. A combined solution is proposed to work out the problems of PA and sub-channel assignment iteratively, and their results indicated that the suggested method outperforms the OMA scheme and prior NOMA schemes by a large margin.

The issue of dynamic power distribution in the multi-cell downlink networks, with each cell using NOMA based resource allocation, is studied in [18]. For users experiencing significant inter-cell interference (ICI), a coordinated multi-point (CoMP) transmission is used. CoMP transmission is used in the CoMP-NOMA architecture when a user is experiencing significant channel gain with several BSs. On the other hand, NOMA is used to schedule both non-CoMP and CoMP users across the same resources. The paper focuses on the joint transmission CoMP-NOMA (JT-CoMP-NOMA). For this paradigm, a distributed power optimization problem with an optimal solution independent of the solutions of other cooperating BSs is provided at each collaborating BS. The results show a remarkable hike in spectral and energy efficiency compared with conventional (CoMP-OMA) networks. 
In [19], the authors have undertaken the task of data rates fairness maximization of various individual users in a multiuser NOMA system. First, they solved the problem for two-user scenarios, where the optimal PA is recovered through the Karush-Kuhn-Tucker (KKT) conditions, whilst the subgradient algorithm is utilized to give the solution of the dual problem. Next, they considered the general multiuser optimization problem in which, under NOMA transmission, the same channel is shared by more than two individual users. Sequential quadratic programming (SQP) was used to solve the nonconvex optimization set problem. It is observed that fairness improves as accessible transmit power increases and decreases as the number of users grows. What is more, it is observed when set side by side to the SQP-based method, power optimization using KKT conditions has a considerably reduced computing complexity.

The influence of PA in round-robin schedules utilizing NOMA systems is evaluated in [20]. Proportional fairness (PF) scheduling is suggested, with the target of providing high levels of throughput and user fairness while maintaining minimal computational complexity. Firstly, an optimal power distribution algorithm that maximizes the weighted sum rate is designed. Next, only a few user pairings are examined for NOMA multiplexing in three rapid and scalable scheduling and user pairing methods. The methods also analyze scenarios when channel state estimation is imprecise and greater than two users are multiplexed across a single resource block.

In [21], a particle swarm optimization (PSO) based NOMA PA technique is investigated. PSO is a solution-finding algorithm that starts with a random solution initially and works its way to the best possible solution through running multiple iterations. The simulation results indicate that using a PA method based on a PSO can markedly raise the system's overall energy efficiency level. The effect of UP on the performance of fixed power allocated NOMA (F-NOMA) is characterized in [22], as well as a NOMA influenced by cognitive radio. The simulation results demonstrate that F-NOMA can provide a more significant sum-rate than that of OMA, and by choosing users whose channel states are more distinctive, F-NOMA's performance gain over conventional MA can be further magnified. When it comes to CR-NOMA, due to the fact that the transmit power distributed to other users is limited based on the notion of cognitive radio networks, the quality of service (QoS) for users with the relatively lower quality channel state can be ensured. CR-NOMA prefers to match the user whose channel state is the best with the user whose channel state is the second-best, whilst the worst channel state possessing user is preferred to be matched to the best channel state possessing individual user by F-NOMA.

As the primary contribution, the authors of [23] analyzed the optimal PA in NOMA systems with a set of channel assignments over numerous channels and following various performance criteria. The authors considered as criteria the weighted sum-rate maximization, maximum fairness, optimization of energy efficiency with weights or QoS restrictions, and sum-rate maximization involving quality of service (QoS) restrictions. Furthermore, for each individual channel, the order limitations on the powers of the users were also considered. The optimal PA taking into account the considered benchmark in semi-closed or closed-form was then proposed in the article. In addition, the writers put forward an effective strategy for optimizing channel assignment and PA jointly and with a minimal level of complexity.

In [24], the authors first improved the current single-carrier user selection and power control methods' computational complexity in NOMA systems. These schemes are then utilized to design new algorithms. One of the algorithms, OPT-JSPA, calculates an optimal solution; Yet, its time complexity, which is characterized by pseudo-polynomial equations, makes it unfeasible for real-world applications requiring minimal latency. Another algorithm, $\varepsilon$-JSPA, is an approximation approach that is characterized by an entirely polynomial time complexity and stands out by balancing the tight tradeoff between the assurance of performance and the complexity. The final algorithm they proposed is the GRAD-JSPA and is a gradient descent-based formula. In [25], the authors elaborated the core idea of NOMA in the downlink as well as uplink transmission. They presented their proposed NOMA strategy for the condition in which 
the base station is arrayed with numerous antennas. Their simulation show NOMA exhibits a potential increase, in comparison to OMA, of system-level throughput.

For NOMA systems with the restriction of user fairness, a resource allocation strategy with minimal complexity that provides a balance between energy and spectral efficiency was presented in [26]. A PA method with dual-layer and a user scheduling technique with its complexity in the lower side is explored by the authors in combination. Intra-subchannel PA is handled using the Lagrangian multiplier approach in the inner layer, whereas an iterative technique formed on the bisection approach is suggested in the outer layer. Simulations indicate that the proposed NOMA system lends higher energy efficiency (EE) and spectral efficiency (SE) while requiring low complexity.

The authors of [27] aim to maximize EE and fairness between users jointly with respect to the subcarrier and power distribution characteristics in downlink NOMA systems. In the paper, it is suggested a greedy subcarrier assignment technique that strictly follows the worst-user first concept. The PA problem is first transformed into a comparable subtractive form and is then solved with the help of fractional programming in which vectors for allocating subchannel power are optimized sequentially. Their proposed algorithms are characterized by their minimal complexity, rapid convergence and insensitivity to the starting conditions.

The authors of [28] proposed solutions for both user pairing and power distribution to optimize the sum SE and EE of the system. The closed-form solutions are derived using the energy efficiency optimizing Dinkelbach (DKL) algorithm, and for maximizing the system sum-rate Karush-Kuhn-Tucker (KKT) conditions were utilized. Furthermore, two users, each with a distinct channel condition, are paired using the Hungarian (HNG) algorithm. The results in the paper reveal that with 20 users included in the system, the suggested optimal PA's sum rate is 6.7 per cent greater than convex programming difference NOMA (NOMA-DC). Similarly, when the proposed optimal PA is used instead of NOMA-DC, the energy efficiency is 66 per cent greater.

The chief contribution of our paper entails improving the total sum rate of NOMA systems and thus maximize the users' data rate to enhance the fairness performance and reduces the outage probability. To achieve our goals, we proposed a UP method based on a simple searching for subcarrier with the minimum average channel gains to be assigned its paired users and then excluding it from the following searching process.

\section{System Model}

We consider a downlink NOMA network, as shown in Fig. 1. The BS is situated in the cell's center area and serves K users uniformly distributed in the cell area. The total bandwidth $B$ is portioned among $S$ subcarriers, with bandwidth $W=\frac{B}{S}$ per subcarrier. The paired users transmit information in the same subcarrier.

For each pair of users $i, j \in\{1, \ldots, K\}$, a superimposed signal is transmitted by the BS on subcarrier $s$ as:

$x_{s}=\sqrt{P_{s, i}} M_{s, i}+\sqrt{P_{s, j}} M_{s, j}$

where $P_{s, i}$ and $P_{s, j}$ are the assigned power to users $i$ and $j$. Moreover, $M_{s, i}$ and $M_{s, j}$ donates the sent signal (i.e., message) to users $i$ and $j$ on subcarrier $s$.

On subcarrier $s$, user k received signal can be written as

$y_{s, k}=h_{s, k} x_{s}+Z_{s, k}$

where $h_{s, k}$ donates the BS's complex channel gain to the $k^{\text {th }}$ user on subcarrier $s$ and $Z_{s, k}$ is the "complex 
additive white Gaussian noise" (AWGN) at user $k$ with zero mean and variance $\sigma^{2}=N_{0} \frac{B}{S}$, where $N_{o}$ is the "noise-power-spectral-density".

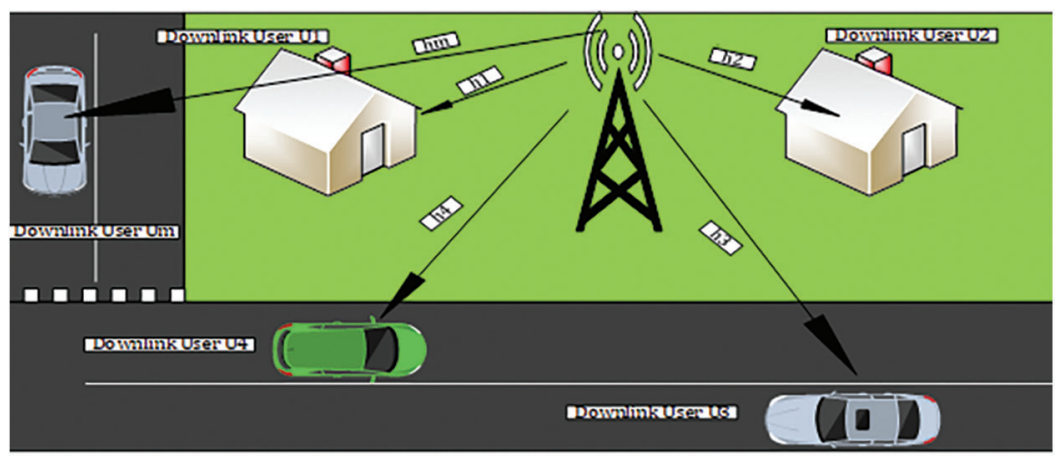

Figure 1: System model for NOMA downlink

The SIC technique is used at the receiver to recover the signal that is wanted. For successful performance of SIC process, the gain of the channel normalized by noise for the user $j \in\{1, \ldots, K\}$ must be less than that of the user $i \in\{1, \ldots, K\}$ on subcarrier $s$, i.e. $\frac{\left|h_{s, j}\right|^{2}}{\sigma^{2}}<\frac{\left|h_{s, i}\right|^{2}}{\sigma^{2}}$. The receiver of user $j$ can decipher its signal message $M_{s, j}$ directly without utilizing the SIC technique and regards the signal of user $i$ as interference. On the other hand, user $i$ receiver will decode the signal message of user $j$ i.e., $M_{s, j}$ at first, and then extracted it from the received superimposed signal to enable the user $i$ to recognize its signal $M_{s, i}$ devoid of interruption from the user $j$.

To facilitate the SIC process, the BS must provide low-gain users greater power. i.e., $P_{s, i}<P_{s, j}$. By assuming that each subcarrier's bandwidth is normalized and $N_{o}$ is recognized as consistent overall subcarriers, accordingly, the data rate for each pair of users $i, j$ on subcarrier $s$ are represented as follows:

$R_{s, i}=\log _{2}\left(1+\frac{P_{s, i}\left|h_{s, i}\right|^{2}}{N_{0}}\right)$

$R_{s, j}=\log _{2}\left(1+\frac{P_{s, j}\left|h_{s, j}\right|^{2}}{P_{s, i}\left|h_{s, j}\right|^{2}+N_{0}}\right)$

The entire system sum-rate is then calculated as follows:

$R_{T}=\sum_{s=1}^{S} R_{s}$

where, $R_{s}=R_{s, i}+R_{s, j}$ is the subcarrier $s$ overall sum-rate.

\section{The Proposed User Pairing Algorithm}

The proposed algorithm aims to attain the following objectives with accepted computational complexity:

- Enhancing the total sum-rate of NOMA system.

- Enhancing the users' data rate to raise the fairness performance and reduce the probability of outage.

The following are the detailed steps of the suggested UP algorithm: 
1) Supposed the channel gain matrix for subcarriers-users, compute each subcarrier's average channel gain (i.e., the average value of each row).

2) Finding the subcarrier with the lowest average gain of channel

3) Assign the user with the utmost channel gain over the subcarrier in step 2 as the first paired user (i.e., strong user) and the user with the next biggest channel gain over this subcarrier as the second paired user (i.e., weak user).

4) Updating the channel gain matrix for subcarriers-users after excluding the subcarrier of step 2 and its assigned paired users.

5) Repeat all previous steps (i.e., steps 1, 2, 3, and 4) until the completion of UP process over all subcarriers.

The pseudo-code that follows the suggested WSF-SUAA steps is shown in Algorithm 1.

\section{Algorithm 1 Proposed User Pairing Algorithm}

1: Initialization: Formulate channel gain matrix.

$\boldsymbol{H}=\left|\boldsymbol{h}_{\boldsymbol{s}, \boldsymbol{k}}\right|^{2} \forall \boldsymbol{s} \in \boldsymbol{S}$ subcarriers \& $\boldsymbol{k} \in \boldsymbol{K}$ users.

2: $\quad$ for $s=1$ to $S$ do

3: $\quad$ Compute each s subcarrier's average channel gain:

$\boldsymbol{H}_{\boldsymbol{s}}^{\boldsymbol{a v g}}=\boldsymbol{a v g}\left|\boldsymbol{h}_{\boldsymbol{s}, \boldsymbol{k}}\right|^{2} \quad \forall \boldsymbol{s}$.

4: $\quad$ end for

5: $\quad$ Finding the subcarrier with the minimum average channel gain ( $\boldsymbol{H}_{s}^{\text {avg }}$ )

6: $\quad$ Assign the biggest channel gain experiencing user over this subcarrier (step 5) as the first paired user (and the next greatest channel gain experiencing user over this subcarrier as the second paired user.

7: $\quad$ Updating the channel gain matrix for subcarriers-users $\boldsymbol{H}$ after excluding the subcarrier of step 5 and its assigned paired users in step 6.

8: $\quad$ Repeat steps 2-7 until the completion of UP process over all subcarriers.

9: $\quad$ End of the Algorithm.

\section{Simulation Results}

In this part, the simulation results of the proposed-UP algorithm's performance evaluation against "random UP" and "conventional UP" are presented. During the simulations, a Rayleigh distributed "frequency-selective-fading channel" is considered.

A fixed PA is considered to allocate the power to each subcarrier $s$ between its two paired users, hence $0.2 P_{s}$ is assigned to a strong user and $0.8 P_{s}$ is allotted to a weak user, where $P_{s}$ is the power per subcarrier. The simulations are averaged over total of 1000 channel realizations for 16 subcarriers and 32 users.

The total sum rate of the system $v s$. "the signal-to-noise-ratio" (SNR) of each subcarrier is presented in Fig. 2, and it is proven that the proposed UP algorithm attains the highest total sum rate with a significant superiority over the other NOMA algorithms at small SNR. 


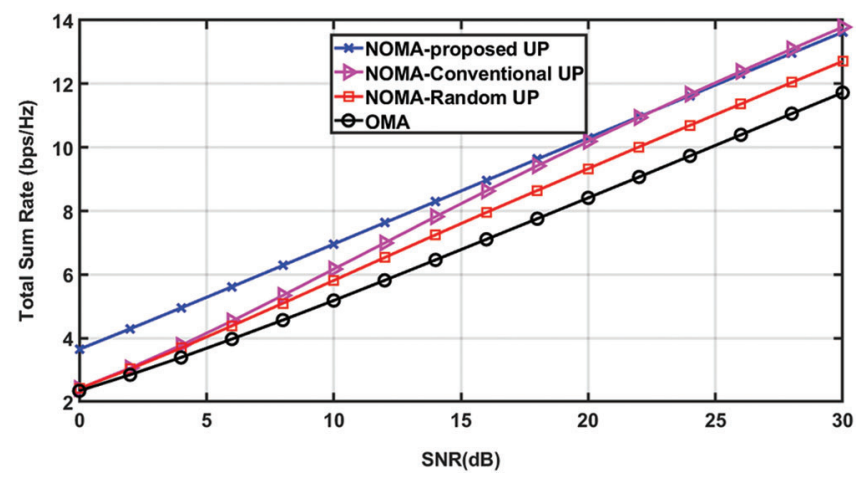

Figure 2: The total sum rate $v$ s. SNR

In Fig. 3, the fairness index (FI) vs. SNR is used to evaluate the fairness performance of the proposed algorithm's user pairing process to that of various UP methods, and it is described in terms of users' data rates as [25]:

$F I=\frac{\left(\sum_{k=1}^{K} R_{k}\right)^{2}}{K \sum_{k=1}^{K}\left(R_{k}\right)^{2}}$

Fig. 3 clarifies that both the proposed UP algorithm and random UP provide a significantly better FI compared to conventional UP, especially at small SNR, and the proposed UP algorithm provides the highest FI for $\mathrm{SNR}<15 \mathrm{~dB}$.

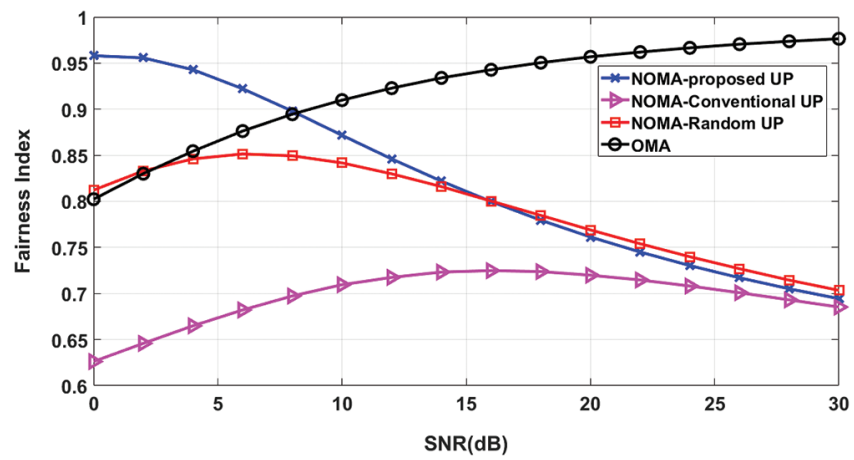

Figure 3: The fairness index (FI) versus SNR

The outage probability, which measures the probability that the user data rate will not reach a specific target data rate, is one of the essential performance indicators. Fig. 4 presents outage probability at target data rate of $1 \mathrm{bps} / \mathrm{Hz}$ versus SNR. It is clearly seen that the proposed UP algorithm provides the lowest outage probability while conventional UP provides the worst outage probability. 


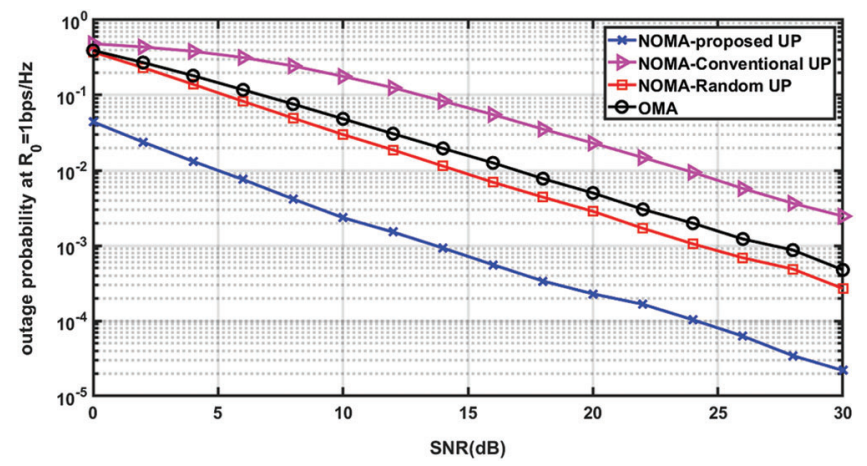

Figure 4: The outage probability at fixed data-rate of $1 \mathrm{~b} / \mathrm{s} / \mathrm{Hz}$ versus SNR

\section{Conclusions}

In this study, we consider the resource allocation problem with respect to user pairing (UP) in downlink non-orthogonal multiple access (NOMA) systems. The proposed UP algorithm is based on the enhancement of the sum rate of the paired users per subcarrier and consequently enhancement of the total sum rate of downlink NOMA systems. The proposed-UP algorithm is based on a simple search for the subcarrier with the minimum average channel gains to be assigned its paired users and then excluding it from the next searching process. The simulation results demonstrate that the suggested UP algorithm not only improves the total sum rate when compared to random UP and conventional UP, but also improves user fairness and minimize the outage probability.

Acknowledgement: The authors would like to acknowledge the support received from Taif University Researchers Supporting Project Number (TURSP-2020/214), Taif University, Taif, Saudi Arabia.

Funding Statement: This research was supported by Taif University Researchers Supporting Project Number (TURSP-2020/214), Taif University, Taif, Saudi Arabia (www.tu.edu.sa).

Conflicts of Interest: The author declares that he has no conflicts of interest to report regarding the present study.

\section{References}

[1] G. Wunder, P. Jung, M. Kasparick, T. Wild, F. Schaich et al., "5GNOW: Non-orthogonal, asynchronous waveforms for future mobile applications," IEEE Communications Magazine, vol. 52, no. 2, pp. 97-105, 2014.

[2] Q. C. Li, H. Niu, A. T. Papathanassiou and G. Wu, "5G network capacity: Key elements and technologies," IEEE Vehicular Technology Magazine, vol. 9, no. 1, pp. 71-78, 2014.

[3] P. Wang, J. Xiao and L. Ping, "Comparison of orthogonal and non-orthogonal approaches to future wireless cellular systems," IEEE Vehicular Technology Magazine, vol. 1, no. 3, pp. 4-11, 2006.

[4] M. I. Kadir, S. Sugiura, J. Zhang, S. Chen and L. Hanzo, "OFDMA/Sc-fDMA aided space-time shift keying for dispersive multiuser scenarios," IEEE Transactions on Vehicular Technology, vol. 62, no. 1, pp. 408-414, 2013.

[5] Z. Zhang, H. Sun and X. Lei, "Non-orthogonal multiple access," in Encyclopedia of Wireless Networks, X. Shen and K. Zhang (Eds.), Cham: Springer International Publishing, chap. 1, pp. 1-4, 2018.

[6] Y. Wang, B. Ren, S. Sun, S. Kang and X. Yue, "Analysis of non-orthogonal multiple access for 5G," China Communications, vol. 13, no. 2, pp. 52-66, 2016.

[7] I. Chih-Lin, S. Han, Z. Xu, Q. Sun and Z. Pan, "5G: Rethink mobile communications for 2020+," Philosophical Transaction Royal Society, vol. 374, no. 2026, pp. 20140432, 2016.

[8] S. M. R. Islam, M. Zeng, O. A. Dobre and K. Kwak, "Nonorthogonal multiple access (NOMA): How it meets 5G and beyond," in Wiley 5 G Ref. R. Tafazolli, P. Chatzimisios and C. Wang (Eds.), New York: John Wiley and Sons, chap. 32, pp. 1-24, 2019. 
[9] Z. Ding, Y. Liu, J. Choi, Q. Sun, M. Elkashlan et al., "Application of non-orthogonal multiple access in LTE and 5G networks,” IEEE Communications Magazine, vol. 55, no. 2, pp. 185-191, 2017.

[10] J. Choi, "Throughput analysis for multiuser diversity of two users with SIC in NOMA systems," in Proc. 2018 Int. Conf. on Signals and Systems (ICSigSys), Bali, Indonesia, pp. 120-124, 2018.

[11] S. Pazhayakandathil, D. K. Sukumaran and A. H. Koodamannu, "Optimal user selection and power allocation for revenue maximization in non-orthogonal multiple access systems," Electronics and Telecommunications Research Institute (ETRI) Journal, vol. 41, no. 5, pp. 626-636, 2019.

[12] Y. Saito, A. Benjebbour, Y. Kishiyama and T. Nakamura, "System-level performance evaluation of downlink nonorthogonal multiple access (NOMA)," in Proc. 2013 IEEE 24th Annual Int. Symp. on Personal, Indoor, and Mobile Radio Communications (PIMRC), London, UK, pp. 611-615, 2013.

[13] J. A. Oviedo and H. R. Sadjadpour, "A fair power allocation approach to NOMA in multiuser SISO systems," IEEE Transactions on Vehicular Technology, vol. 66, no. 9, pp. 7974-7985, 2017.

[14] M. Abd-Elnaby, G. G. Sedhom and M. Elwekeil, "Subcarrier-user assignment in downlink NOMA for improving spectral efficiency and fairness," IEEE Access, vol. 9, pp. 5273-5284, 2021.

[15] L. Chen, L. Ma and Y. Xu, "Proportional fairness-based user pairing and power allocation algorithm for nonorthogonal multiple access system," IEEE Access, vol. 7, pp. 19602-19615, 2019.

[16] J. E. C. Junior, M. S. Alencar, J. V. dos S. Filho and K. D. R. Assis, "A fair power allocation for non-orthogonal multiple access in the power domain," The ISC International Journal of Information Security, vol. 11, no. 3, pp. 123-130, 2019.

[17] B. Di, L. Song and Y. Li, "Sub-channel assignment, power allocation, and user scheduling for nonorthogonal multiple access networks," IEEE Transactions on Wireless Communications, vol. 15, no. 11, pp. 7686-7698, 2016.

[18] M. S. Ali, E. Hossain, A. Al-Dweik and D. I. Kim, "Downlink power allocation for CoMP-nOMA in multi-cell networks," IEEE Transactions on Communications, vol. 66, no. 9, pp. 3982-3998, 2018.

[19] Z. Ali, G. A. S. Sidhu, M. Waqas and F. Gao, "On fair power optimization in non-orthogonal multiple access multiuser networks," Transactions on Emerging Telecommunications Technologies, vol. 29, no. 12, pp. e3540, 2018.

[20] J. He, Z. Tang, Z. Tang, H. Chen and C. Ling, "Design and optimization of scheduling and non-orthogonal multiple access algorithms with imperfect channel state information," IEEE Transactions on Vehicular Technology, vol. 67, no. 11, pp. 10800-10814, 2018.

[21] Y. Guo and H. Li, "A power allocation method based on particle swarm algorithm for NOMA downlink networks," Journal of Physics: Conference Series, vol. 1087, no. 2, p. 022033, pp. 1-7, 2018.

[22] Z. Ding, P. Fan and H. V. Poor, "Impact of user pairing on 5G non-orthogonal multiple-access downlink transmissions," IEEE Transactions on Vehicular Technology, vol. 65, no. 8, pp. 6010-6023, 2016.

[23] J. Zhu, J. Wang, Y. Huang, S. He, X. You et al., "On optimal power allocation for downlink non-orthogonal multiple access systems," IEEE Journal on Selected Areas in Communications, vol. 35, no. 12, pp. 27442757, 2017.

[24] L. Salaun, M. Coupechoux and C. S. Chen, "Joint subcarrier and power allocation in NOMA: Optimal and approximate algorithms," IEEE Transactions on Signal Processing, vol. 68, no. 1, pp. 2215-2230, 2020.

[25] K. Higuchi and A. Benjebbour, "Non-orthogonal multiple access (NOMA) with successive interference cancellation for future radio access," IEICE Transactions on Communications, vol. E98.B, no. 3, pp. 403-414, 2015.

[26] Y. Zuo, X. Zhu, Y. Jiang, Z. Wei, H. Zeng et al., "Energy efficiency and spectral efficiency tradeoff for multicarrier NOMA systems with user fairness," in Proc. 2018 IEEE/CIC Int. Conf. on Communications in China (ICCC), Beijing, China, pp. 666-670, 2018.

[27] A. J. Muhammed, Z. Ma, P. D. Diamantoulakis, L. Li and G. K. Karagiannidis, "Energy-efficient resource allocation in multicarrier NOMA systems with fairness," IEEE Transactions on Communications, vol. 67, no. 12, pp. 8639-8654, 2019.

[28] Z. J. Ali, N. K. Noordin, A. Sali, F. Hashim and M. Balfaqih, "Novel resource allocation techniques for downlink non-orthogonal multiple access systems," Applied Sciences, vol. 10, no. 17, p. vol. 5892, pp. 1-19, 2020. 